This is the author's final, peer-reviewed manuscript as accepted for publication. The publisher-formatted version may be available through the publisher's web site or your institution's library.

\title{
Load shedding strategies for preventing cascading failures in power grid
}

S. Pahwa, C. Scoglio, S. Das, N. Schulz

\section{How to cite this manuscript}

If you make reference to this version of the manuscript, use the following information:

Pahwa, S., Scoglio, C., Das, S., \& Schulz, N. (2013). Load shedding strategies for preventing cascading failures in power grid. Retrieved from http://krex.ksu.edu

\section{Published Version Information}

Citation: Pahwa, S., Scoglio, C., Das, S., \& Schulz, N. (2013). Load shedding strategies for preventing cascading failures in power grid. Electric Power Components and Systems, 41(9), 879-895.

Copyright: @ Taylor \& Francis Group, LLC

Digital Object Identifier (DOI): doi:10.1080/15325008.2013.792884

Publisher's Link:

http://www.tandfonline.com/doi/full/10.1080/15325008.2013.792884\#.Uh-pZ9gpjsQ

This item was retrieved from the K-State Research Exchange (K-REx), the institutional repository of Kansas State University. K-REx is available at http://krex.ksu.edu 


\title{
Load shedding strategies for preventing cascading failures in power grid
}

\author{
S. Pahwa*, C. Scoglio, S. Das, N. Schulz \\ Electrical and Computer Engineering Department \\ Kansas State University \\ Manhattan, KS 66506-5204, USA
}

\begin{abstract}
Load shedding has always been a commonly adopted method in emergency situations to maintain power system reliability. Several load reduction strategies have been suggested in the past but most are complex and not scalable. In this paper, we have proposed and thoroughly investigated three load shedding strategies to prevent cascading failures in power grid. The first strategy is a base line case called the homogeneous load shedding strategy. It reduces load homogeneously in all the buses of the system. This strategy is extremely simple and fast, and these properties motivate its use in some specific scenarios in spite of its inefficiencies. Next, to accurately find the location and amount of load shedding, we propose a linear optimization formulation which is much more efficient in overall load shedding in the system. A novel tree heuristic is proposed to overcome the drawbacks of the optimization, namely fairness and scalability. The tree heuristic is linear and very simple to implement. In general, it gives close to optimal results. The results of the tree strategy are compared with that of another existing heuristic and it is found that the tree performs equal to or better than the existing heuristic for all cases.
\end{abstract}

\section{Index Terms}

Cascading failures, Mitigation Strategies, Load shedding, Homogeneous Load Reduction, Optimal Load Reduction, Tree Heuristic

* Corresponding author

Email addresses: sakship@ksu.edu (S. Pahwa), caterina@ksu.edu (C. Scoglio), sdas@ksu.edu (S. Das), noels@ksu.edu (N. Schulz) 


\section{INTRODUCTION}

Modern technology has led to the use of huge amounts of electricity in the present era. It would not be wrong by any means to say that the usage of electricity has become inevitable in every walk of our lives. The existing grid was designed years ago keeping in mind such increase in its usage in the years to come. However, the time has come when the existing grid requires expansion, improved technology, better security and restructuring to accommodate the ever increasing demand. With the advent of the smart grid, a lot of these concerns will be addressed. Besides, extensive research is being carried out in industry and academia to address these issues. North American Electric Reliability Corporation (NERC) defines a cascading failure as "The uncontrolled loss of any system facilities or load, whether because of thermal overload, voltage collapse, or loss of synchronism, except those occurring as a result of fault isolation" [1].

In power grid, flow dynamics depend greatly on the electrical characteristics such as the voltages, impedances, and the difference in the phase angles of a given pair of buses between which the transmission line is present. The system instability can be detected by monitoring one or more of the system quantities such as a sudden change in power flow through specific transmission lines, change of bus voltage angles, etc [2]. If a single line gets overloaded or trips, its power can be immediately re-routed to a different line and the disturbance may be suspended. But sometimes, the other line is also already overloaded and must re-route its increased load to its neighbors. This redistribution of power may lead to the subsequent overloading of other lines causing their malfunction at the same time and the consequence could be a cascade of overloading failures.

Such incidents have taken place in the recent past, like the 1996 blackout in the Western states [3], [4] and the Northeastern blackout of 2003 [5]. Electric power systems collapsed in Denmark, Italy, and the United Kingdom within weeks or months of the 2003 U.S. blackout [6]. Very recently in 2012, India faced a grid collapse which affected 600 million people and was pronounced as the largest blackout in global history in terms of the number of people affected [7].

Large-scale blackouts are due to the concurrent malfunction of a number of transmission lines and power generators often triggered by an initial failure or failures, such as the breakdown of a power line. This is discussed in [8] with the help of a hidden failure model in which some components of the system such as relays have defects that remain dormant until abnormal operating conditions are reached. The authors of [9] have shown that if a line $L$ which shares a bus with other lines fails, the hidden failures in all the other lines sharing the bus are exposed.

If a system instability is detected, sometimes it is necessary to shed selected loads to balance generation and load. Sometimes this load shedding helps to maintain stability without the need for system separation or islanding [2]. The use of load shedding as a mitigation technique has been evolving since several years and different approaches have been proposed in the past [10], [11], [12]. 
In this paper, we present three load reduction strategies and their thorough analysis, to mitigate cascading failures in power grid: Homogeneous load shedding strategy, linear optimization, and tree heuristic. The homogeneous load reduction strategy is a very simple method for executing load shedding and can be used for some very specific and extreme emergency situations in spite of its limitations. It reduces an equal percentage of load in all the buses of the system, making it fair in its execution. At the same time, it is linear and shows low complexity even for large systems. However, since it sheds load in all the buses, the overall load shedding for this strategy is much higher than the other two strategies discussed further. The optimization formulation uses a linear programming technique with an objective to satisfy as much demand in the system as possible. However, the optimization sheds all or a large percentage of load in very few buses to achieve this objective. Thus, it is not fair in the way it sheds load in the system. Also, although polynomial in complexity, it becomes inefficient for large system sizes. As a result, we propose a novel heuristic called the tree heuristic. This heuristic has the same objective as the optimization, that is to satisfy as much demand in the system as possible but it does so in a fair way. It sheds an equal percentage of load in a small part of the system using a tree, and it is found that the buses used for load shedding by the optimization are always a subset of this tree. It is linear in complexity and is more efficient for large systems. The tree heuristic, in general, gives near optimal results. This has been validated numerically as well as graphically by plotting the result of the tree heuristic against an optimal curve. We also verified that the tree heuristic is better in performance than another local load shedding heuristic proposed in literature. These results are discussed later in the paper. However, there are certain cases when the tree heuristic cannot give a solution, based on the system elements that are used to determine the tree. We discuss such cases and give some critical insights on the role of system elements later in the paper. In summary, the following are the main contributions of this paper:

- A novel, efficient and fair heuristic for load shedding

- Critical insights on the impact of network elements on the cascading process

Each of the load reduction strategies has been tested on the 14-, 30-, 57-, 118- and 300-bus IEEE test systems available at [13]. We compare the three strategies on the basis of running time complexity, number of buses involved in load reduction, fairness in load reduction, overall load reduction and the number of cases in which the strategy is effective. Results have been presented in the form of graphs and tables to show the merits and demerits of each strategy.

The paper is organized as follows: Section II describes the previous work that has been done in this field. In Section III, we present the homogeneous load reduction strategy as the base line strategy. In section IV, we describe and explain the optimization formulation with the advantages and drawbacks. Further, in Section V, we propose the tree heuristic, describe the algorithm for its implementation and its merits over the optimization along with its disadvantages. We also show the result graphically with 
respect to the optimal curve and then go on to compare the performance of the tree strategy with another heuristic presented in [14]. Results, and Conclusions, along with future work are presented in Sections VI and VII, respectively.

\section{RELATED WORK}

In the past, researchers have analyzed different mitigation strategies for blackouts in power grids. The authors of [15] have mentioned that their findings suggest, counter intuitively, that sometimes sensible attempts to mitigate failures in complex systems like power grids can have adverse effects and therefore must be approached with care. The authors have considered three types of mitigation measures and evaluated their impact on the frequency of large and small size blackouts. They suggest that mitigation measures, such as reducing the probability that an overloaded line suffers an outage, requiring a certain minimum number of lines to get overloaded before an outage occurs or increasing the generation margin shifts the dynamic equilibrium of the system and brings it to a point of selforganized criticality, reducing the risk of large blackouts. Similar analysis has been carried out in [8]. However, [16] has two other methods of mitigating cascading failures, namely survivability and reciprocal altruism. Besides these newly proposed strategies, load shedding has been a classical and a quite reliable mitigation strategy. There have been several discussions on optimal load shedding in literature recently and in the past [10], [12], [17], [18], [19], [20], [21], [22]. [17] proposes a load shedding method in steps with an equal frequency spacing between steps with an aim to avoid over shedding. [10] shows a non-linear optimization formulation for load shedding during emergencies with distributed generation. They have considered the complexity that arises when distributed generators are included in the system. The authors of [20] propose an optimization problem for load reduction with relaxed constraints. They are of the view that if we allow the voltage to go below its minimum limit and the power flow to go beyond the capacity of the transmission lines for a very short duration, shedding of few loads would be sufficient to mitigate the problem and some of the load cuts can be prevented. [19] use a linear optimization formulation to study load shedding but as two sub-problems. The first sub-problem aims at maximizing the voltage magnitudes of weak buses while the second sub-problem addresses standard load shedding incorporating technical and economic effects. [21] deals with an adaptive load shedding scheme in which the load shedding is adapted to suit the size and location of the experienced disturbance using the local frequency rate information. There has been more recent work on adaptive and combinational load shedding by a lot of researchers. Different methods for adaptive load shedding are adopted in [23], [24], [25], [26], [27], [28]. The authors of [18] talk about interruptible and un-

interruptible parts of every load and suggest shedding the interruptible part of every load in the system as a mitigation strategy.

Most of the above strategies are non-linear and might be difficult to use during emergency situation. 
Moreover, as the system size increases, complexity increases and there might be some convergence problems. As a result, we propose the following three strategies on load reduction: Homogeneous load shedding, optimal load reduction and tree load shedding heuristic. These strategies are discussed in details in the following sections.

\section{Homogeneous LoAd Shedding StRATEgy}

The homogeneous load shedding is a base line load shedding strategy and very simple to implement. This strategy reduces load at all buses in the system by an equal percentage after the initial failure takes place. The percentage of load shedding depends on the location of the initial failure and how critical it is to the system. This can be decided in the planning stages of the system and all the information can be known to the operator previously so that immediate action can be taken when needed. Obviously, this strategy always works and is linear in complexity but the overall load shedding by this strategy is much higher than the other two strategies discussed further, for the simple reason that it reduces load at all buses without determining any specific locations. Thus, is it very simple and quick but at the same time inefficient. We would like to determine quantitatively the amount of additional load shedding required by this strategy to stop a cascade after the initial failure takes place. Results for this strategy and its comparison with the other strategies are discussed in the following sections.

The power flow calculations for each strategy is based on the DC power flow model. During emergency situations, quick and simple techniques are needed to control the unwanted situation and even though less accurate, the DC power flow model serves the purpose in such situations. At the same time, the DC model is linear and does not face any convergence issues. In the past, the DC model has been used to study various problems [29], [30], [31].

\section{OPTIMIZATION FORMULATION}

After the initial failure, there is a need to redistribute the power that was flowing through the failed line, among the neighboring lines. However, since each of the lines have a limit to which they can carry additional power, which is known as the capacity of the lines, care must be taken to redistribute power so that none of the other lines are overloaded. In other words, we need to prevent any other failures due to the initial failure. As a result, sometimes there is a need for load reduction on the buses. However, it is important to know the correct location and amount of load reduction that would solve the purpose, unlike the homogeneous strategy in which even buses which might not be affected by the initial failure at all have to undergo load reduction.

The objective of the optimization formulation is to satisfy as much demand in the system as possible and if there is a need to shed load then find the optimal location and amount for load reduction. It 
considers the presence of multiple generators in the system. The optimization is based on the DC power flow model [32], [33] and uses linear programming techniques.

The problem description is as follows:

Given:

- Topology

- $N$ - number of buses (Loads and generators)

- $L$ - number of lines

- $X$ - inductance of the lines

- $C$ : Capacity of the lines

- $\Lambda_{\text {init }}$ : Initial load and generation vector

- $i-j$ : The failed line

\section{Decision Variables:}

- $\Lambda$ : The new load and generation vector, after load reduction

- $\delta$ : Phase angles

Objective Function:

$$
\operatorname{Max} \sum_{i=1}^{N} \lambda_{i}
$$

where $i \in d, d$ is the set of loads from $\Lambda$

Subject To:

$$
\begin{gathered}
P_{i j}=\frac{\delta_{i}-\delta_{j}}{X_{i j}} \\
P_{i j} \leq C_{i j} \\
\lambda_{i}=\sum_{j=1}^{N} P_{i j} \\
\delta_{\text {slack }}=0 \\
0 \leq \lambda_{i} \leq \lambda_{\text {init }_{i}}
\end{gathered}
$$

where $i \in d$

$$
0 \leq \lambda_{j} \leq 1.05 * \lambda_{\text {init }_{j}}
$$


where $j \in N-d, N-d$ is the set of generators

$$
\sum_{i=1}^{N} \lambda_{i}=0
$$

As seen from the objective function, the optimization aims at maximizing the amount of load satisfied, or in other words, minimize the load shedding. The first constraint is the main equation for the DC power flow model in which the power flow on a line is a function of the phases of the two buses and the inductance of the line between them. The second constraint makes sure that the power flow on every line never exceeds the capacity of the line. This prevents failures due to overloading when redistribution of power takes place after the initial failure. The third constraint is the basic Kirchoff's law which indicates that the algebraic sum of the incoming and outgoing power at any bus must be zero. The fourth constraint ensures that the phase angle of the slack or the reference bus is always zero. The next two constraints give the bounds on the satisfied load for each bus and the available generation and the last constraint says that the sum of loads and generators must be zero, indicating a generation-load balance.

We tested the optimization formulation on the IEEE 14-, 30-, 57-, 118- and 300-bus test systems for all lines in each system. As mentioned before, the optimization solution is based on linear programming and uses either Simplex or a variant of Simplex algorithm. The average running time complexity of the Simplex algorithm is polynomial, precisely $O\left(N^{3}\right)$. In the worst case, Simplex has an exponential complexity given by $2^{N}$. Although the number of buses involved in load reduction are very few, a large percentage of their load, sometimes all of it is shed in order to provide for the other buses. Thus, it is not fair to fulfill the requirements of others at the expense of a few buses.

Considering these drawbacks, we propose a heuristic called the tree load reduction strategy for the optimization formulation. The tree heuristic is described in the next section.

\section{TREE LOAD REDUCTION HEURISTIC}

Similar to the objective of the optimization, this strategy also aims at satisfying as much demand as possible for all buses in the system. It considers load reduction by equal amount on a small subgraph of the system, which is a tree, formed by buses and lines that might be directly or indirectly affected by the re-routing of power due to the initial failure. However, it must be noted that this is not just a forwarding tree but a directional tree carrying the maximum power from the root to the end buses as compared to any other tree starting from the same root. The tree is formed on the demand side of the failure with the root being the demand side bus of the initially failed line. It then propagates until it encounters a generator, a peripheral bus or a sink bus. Once the tree for the given failed line is discovered, an equal percentage of load is reduced on every bus in the tree. Thus, the tree strategy exercises fairness in its execution unlike the optimization. Also, the average running time as well as the worst case complexity of the tree heuristic is $O(N)$. The set of buses that form the tree for a particular failure and the amount 
of load shedding to be carried out on this tree to completely protect the network from further failures can be decided in the planning stages and be known to the operators, making it possible to implement it in real-time. We observed that the buses selected for load shedding in the optimization are always a subset of the buses selected in the tree. However, this fairness in load shedding for all buses in the tree might not be effective in all cases and therefore, this heuristic does not work for every line in the system. The important thing to note, however, is that in all the cases that this strategy works, the amount of overall load shedding is equal to or slightly higher than the overall load reduction of the optimization. For more clarity, we plotted an optimal curve for a given link of the 14-bus system by varying the lower bound on the load satisfaction constraint, from 0 to 1 in steps of 0.1 and also plotted the result of the tree strategy against this curve as shown in Figure 1. The difference between the optimal and the tree value is only $2.64 * 10^{-3}$. The optimal curve and result for the tree strategy for a given failure of the 300-bus system is also plotted in Figure 2.

The algorithm for discovering the tree is described further.

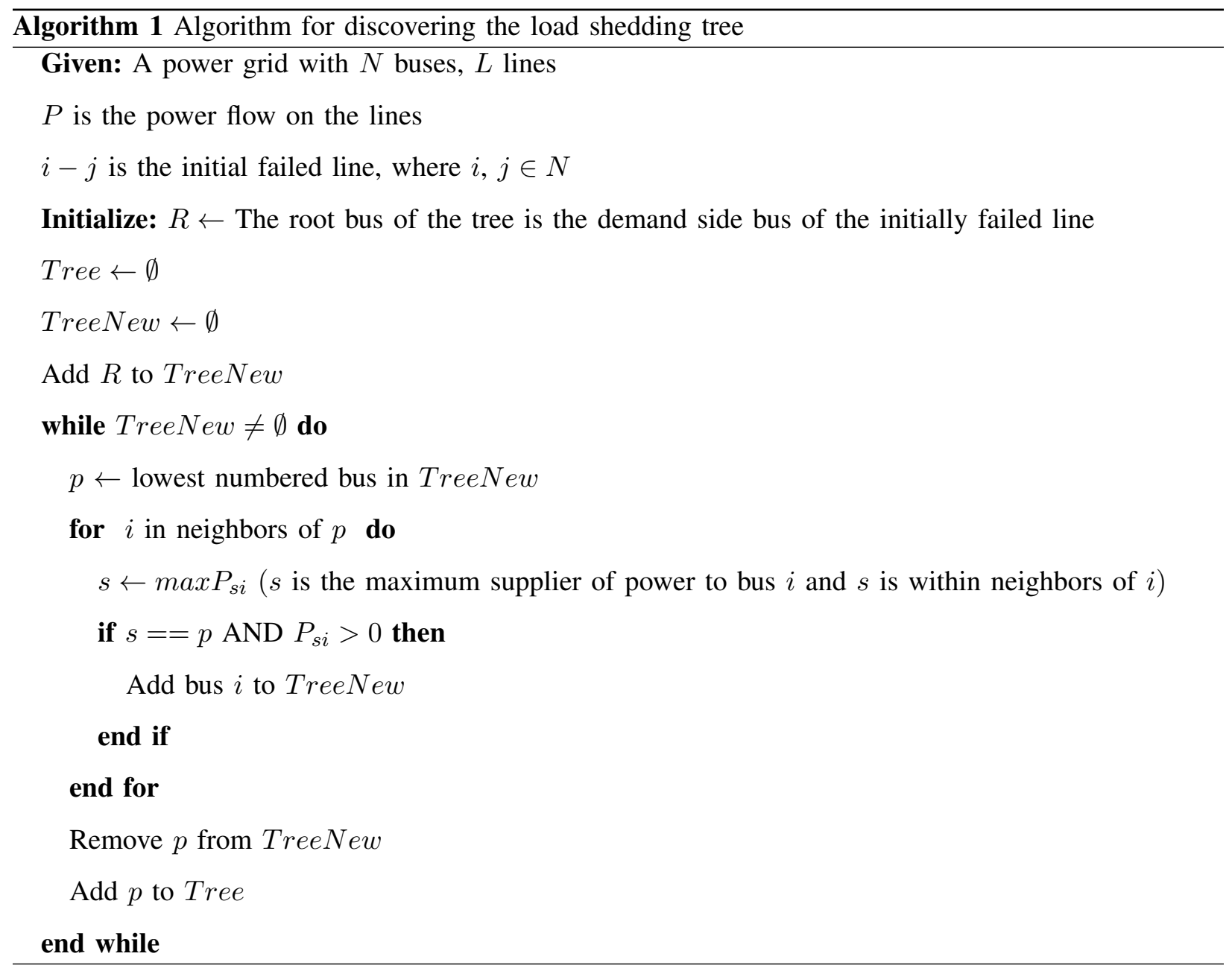


After the tree is discovered, an equal percentage of load is reduced on all the load-carrying buses of the tree. However, there are times when the tree obtained by this process is very small and the buses forming the tree do not carry enough load such that an equal reduction of load on all the buses of the tree can give a solution that will keep the system intact. In some other cases, the maximum power transfer tree consists of buses which are mostly transit buses that help in the transfer of power from the failed line to the other parts of the system but they themselves carry very little or no load. So load shedding on the tree in this case will not help to stop the cascade. In some particular cases, there might be a need to shed an additional amount of load in a particular bus than equal load shedding on all the buses of the tree. In such cases, even a high percentage of load shedding might not be able to avoid the overloading of a few lines around the initial failure and there might be a need to allow for the failure of a few overloaded elements in order to preserve the remaining system. At such times, the optimization is a better load shedding strategy. Thus, we can conclude that the tree heuristic, although quite efficient when it works, is unable to give solutions for all the possible line failures in the system. Most importantly, the amount of load carried by all the buses of the tree affects the working of this strategy.

For the IEEE systems that we have considered, the tree heuristic works for $80 \%$ of the cases for the 14-bus system, $61 \%$ cases for the 30 -bus system, $61 \%$ cases for the 57 -bus system and $59 \%$ cases for the 118- and 300-bus systems.

The Figure 1 shows the result of the tree strategy against the optimal curve for line 3 of the 14-bus system. The x-axis shows the percentage of maximum allowable load shedding for all the nodes of the system after the failure of the initial line 3. The y-axis represents the total load served in the system. The blue stars represent the optimal curve while the red square represents the result of the tree strategy. The difference between the result of the tree strategy and the corresponding point on the optimal curve is only $2.64 * 10^{-3}$. Similarly, the result for line 185 of the 300 -bus system is shown in Figure 2 . The analysis was carried out for all the lines of all the test systems and similar results were seen.

The Figure 3 shows the comparison between the optimization and the tree heuristic for all the links in the 118-bus system. The $\mathrm{x}$-axis represents the ratio of overall load shedding for all the working cases of the 118-bus system. The y-axis represents the percentage of lines. As seen from the histogram, approximately $70 \%$ of the working cases have exactly the same overall load shedding by the tree heursitic as by optimization. The other thing to note here is that for the remaining $30 \%$ of the cases, the load shedding by the tree heuristic is around the optimal except for a very few cases when it strays away from the optimal.

\section{A. Comparison with a local load shedding heuristic}

The heuristic approach to local load shedding is presented in [14]. For simplicity, we will call it heuristic 2. In this heuristic, the node set for load curtailment is found based on the forward and reverse 
power flow tracing in the vicinity of the overloaded line. The amount of load curtailed at each bus is in proportion of load at that bus to the total load considered for load shedding. Low computation time and simplicity are the merits of heuristic 2 . However, the drawback is that sometimes it gives results that are quite far from optimal.

We implemented heuristic 2 for all the five systems and compared the results of the tree strategy with this approach. We found that in some cases the tree strategy preformed much better than heuristic 2 and at other times the amount of load shedding was equal for both. Also, the tree strategy worked for more cases than heuristic 2 did but for all the cases that the tree strategy does not work, heuristic 2 also does not work. The Table I shows the results for the different load shedding methods discussed so far for all 20 lines of the 14-bus system. It is considered that each line fails, one at a time and is known as the initial failure. All the loads are in per unit. The value under each load shedding strategy is the percentage of overall load shedding by the strategy for the given line failure. These results are also shown by the graph in Figure 4. The x-axis represents the line identification and the y-axis represents the percentage of overall load shedding. For some lines, the load shedding bars for one or both heuristics is missing but that of the optimization is present, indicating that the heuristic/s fails to work for that particular line. For some other lines, the bar for the optimization is also missing along with that of the heuristics. These are the cases when no load shedding is required upon the failure of that particular line since it does not cause overloading of any other lines upon its failure.

\section{B. Comparison with the homogeneous load shedding strategy}

The Figure 5 represents the overall load shedding when each line of the 14-bus system fails, one at a time. The $x$-axis represents the line id while the $y$-axis represents the percentage of total load shedding in the system. As discussed before, the tree heuristic does not work for all the cases. For the 14-bus system, the tree heuristic works for $80 \%$ of the lines in the system and so the graph shows the results for those $80 \%$ cases. Certain lines show a load shedding of $0 \%$ which means they do not require any mitigation upon their failure. This is possible if there is enough buffer capacity in the neighboring lines of this failed line to re-route the additional power or this line was not carrying a heavy load in the very first place. Similarly, the Figure 6 represents the overall load shedding for the 118-bus system. For clarity, only a sample of the lines for the 118-bus system are shown. The results and comparisons are discussed in more detail in the next section.

The results show that the homogeneous strategy reduces a large percentage of load as compared to the other strategies. However, due to its simplicity and linearity, it can be useful to mitigate cascades in situations when it is much more important to implement a remedy rather than to check the efficiency of it. As seen from the graphs, as long as the homogeneous strategy mitigates the cascade with up to $50 \%$ load shedding, it can be useful in the absence of the other strategies. The table II shows the general 
comparison between the three strategies.

\section{RESUlTS}

Figures 1, 2 and 3 show the comparison between the optimization and the tree heuristic with the help of the optimal curve and the histogram. The optimal curve shows the result for a single line of the 14-bus system whereas the histogram shows the result for all the lines of the 118-bus system. The histogram shows that the tree heuristic works efficiently, in general, for the larger systems also.

Table I and Figure 4 show the comparison of the overall load shedding for all the 20 lines of the 14bus system among optimization, tree heuristic and heuristic 2, a local load shedding heuristic previously implemented in [14]. These comparisons clearly validate the efficiency of the tree strategy and its ability to give near-optimal results for all the working cases.

Table II shows a general comparison between the three load shedding strategies. It is seen that the optimization sheds the minimum percentage of overall system load among the three strategies. However, it is not fair in the way it sheds load; it sheds an excessive percentage of load for a few buses and has a polynomial running time. Considering these drawbacks of the optimization, we proposed the tree heuristic which implements fairness in its execution and has a linear running time complexity. However, we found that this fair load shedding for a larger number of buses might not be the most efficient strategy in every case. There might be failures which are more severe than the others and in such cases a larger percentage of concentrated load shedding might be a better option. The homogeneous load shedding heuristic is a base line heuristic and reduces an equal percentage of load in all the buses of the system. In times of emergency, this might be a very quick strategy to implement even for large systems. However, the overall percentage of load shedding required by this strategy is much higher than the other two strategies.

Table III shows three representative cases for each of the IEEE 14-, 30-, 57-, 118- and 300-bus systems. In the table, the three cases for each system were picked from the set of cases for which the tree heuristic was able to keep the entire system intact without losing a single additional element after the initial failure. There were several cases in which the tree heuristic could save the entire system besides one or two additional failures but these cases were not a fair comparison with the optimization and homogeneous strategy which preserve the entire system at all times and so were not included in the analysis. The table shows the number of buses involved in load shedding and the total overall load shed for the representative cases of each system, for the three strategies.

The optimization and the tree heuristic are very close in their performance but quite different in the initial set-up and implementation. The homogeneous is very simple in both initial set-up as well as implementation and can be used in extreme emergency situations but it is a very basic strategy and not a very efficient load reduction technique.

Figure 5 shows the comparison of the three strategies for the 14-bus system. The $\mathrm{x}$-axis represents 
the line id for the set of lines for which the tree heuristic works. 16 out of 20 possible cases are in the working set for the 14-bus system. The y-axis represents the percentage of total load shed of the system when a particular line is removed as the initial failure. The figure shows that the tree heuristic performs very close to the optimization whereas the homogeneous heuristic causes an excessive load shed in the system for the same initial failure. There are certain lines for which no mitigation in the form of load shed is needed and these are the non-critical lines. Figure 6 shows a comparison of the strategies for a sample of the working cases for the 118-bus system. Only a sample of the working cases and not all the cases were chosen for reasons of clarity because there were too many cases to represent on a single graph. However, this figure shows that the homogeneous strategy performs poorly as the system size goes on increasing.

The three load shedding strategies were tested thoroughly for all the five systems and we observed similar trends in the other systems too.

\section{CONCLUSIONS AND Future WORK}

The optimization formulation is a very effective strategy for load shedding since it works in every case where a mitigation strategy is needed and helps to keep the system intact with very little overall load shedding. However, it is not fair in its execution and reduces a large percentage of load on one or very few buses while keeping the load on all the other buses as it is. Thus, it works for the benefit of almost all the customers at the cost of very few customers. At the same time, as the system size increases, the optimization becomes computationally more expensive. This leads us to propose the tree heuristic for the optimization. The tree heuristic works on the same objective as the optimization, that is to satisfy as much load as possible. However, it works on a fair basis to reduce an equal percentage of load on all the load-carrying buses of the tree and is always linear in its operation. We observed that the buses selected for load shedding by the optimization are always a subset of the buses in the tree for any given initial failure. The equal load reduction sometimes does not help to keep the system intact and causes overloading of some neighboring lines. Thus, the tree heuristic does not work in all the cases but in the majority of the cases that it works, its performance is equivalent to the optimization formulation. The Homogeneous heuristic is the base line strategy; it is very simple to implement and is not expensive computationally. However, it is not a very efficient strategy because it reduces an equal percentage of load on all buses of the system, irrespective of whether it is required for a given bus or not. Thus, although not expensive in computation, it is a very expensive strategy in implementation. However, it might be a reliable strategy in times of emergency when there is not enough time. In general, the optimization and the homogeneous strategies are more centralized in nature whereas the tree heuristic is implemented based on local information and is more distributed in nature. 
Our future work includes proposing an optimal islanding scheme as well as heuristics for the same as a mitigation strategy. There is some work discussed in [34] for optimal islanding. Although these islands are optimal, it is usually not a scalable problem. Hence the need to propose heuristics for the same.

\section{ACKNOWLEDGMENTS}

This work was supported by the Electric Power Affiliates Program, consisting of Westar Energy, Burns and McDonnell, Omaha Public Power District, and Nebraska Public Power District.

The authors would like to thank A. Pahwa and P. Schumm for their valuable suggestions and contributions to this work. 
TABLE I

COMPARISON OF PERCENTAGE OF OVERALL LOAD SHEDDING AMONG THE OPTIMIZATION, TREE HEURISTIC AND HEURISTIC 2 FOR ALL 20 LINES OF THE 14-BUS SYSTEM

\begin{tabular}{|c|c|c|c|c|}
\hline Strategy & Bus connections & Optimization & Tree heuristic & Heuristic 2 \\
\hline \multicolumn{5}{|l|}{ Line Id } \\
\hline 1 & $1-2$ & 46.95 & N/A & N/A \\
\hline 2 & $1-5$ & 12.94 & 14.16 & 14.47 \\
\hline 3 & $2-3$ & 24.43 & 24.61 & 24.61 \\
\hline 4 & $2-4$ & 3.83 & 5.12 & 5.2 \\
\hline 5 & $2-5$ & 0 & 0 & 0 \\
\hline 6 & $3-4$ & 0 & 0 & 0 \\
\hline 7 & $4-5$ & 12.70 & 17.06 & 42.71 \\
\hline 8 & $4-7$ & 8.62 & 9.45 & 9.94 \\
\hline 9 & $4-9$ & 2.73 & 3.82 & 13.24 \\
\hline 10 & $5-6$ & 15.13 & N/A & N/A \\
\hline 11 & $6-11$ & 1.35 & 1.36 & 2.12 \\
\hline 12 & $6-12$ & 1.66 & 1.67 & N/A \\
\hline 13 & $6-13$ & 11.65 & N/A & N/A \\
\hline 14 & $7-8$ & 0 & 0 & 0 \\
\hline 15 & $7-9$ & 8.62 & 9.45 & 13.24 \\
\hline 16 & $9-10$ & 2.02 & 2.05 & 2.05 \\
\hline 17 & $9-14$ & 3.13 & 3.14 & 3.14 \\
\hline 18 & $10-11$ & 0 & 0 & 0 \\
\hline 19 & $12-13$ & 0 & 0 & 0 \\
\hline 20 & $13-14$ & 1.48 & 1.49 & 1.5 \\
\hline
\end{tabular}


TABLE II

GENERAL COMPARISON BETWEEN THE THREE LOAD REDUCTION STRATEGIES

\begin{tabular}{|c|c|c|c|}
\hline Strategy & Optimization & Tree & Homogeneous \\
\hline \hline Avg. complexity & $O\left(N^{3}\right)$ & $O(N)$ & $O(N)$ \\
\hline Buses involved & Very few & Few & All \\
\hline Total reduction & Very small & Very small & Large \\
\hline Fairness & Not fair & Fair & Fair \\
\hline
\end{tabular}

TABLE III

COMPARISON OF THE THREE STRATEGIES WITH SOME REPRESENTATIVE CASES FROM EACH OF THE TESTED SYSTEMS

\begin{tabular}{|c|c|c|c|c|c|c|c|}
\hline Sys & Bus conn & \multicolumn{2}{|c|}{ Optim. } & \multicolumn{2}{|c|}{ Tree } & \multicolumn{2}{|c|}{ Homog. } \\
\hline & & Bus & Tot.red & Bus & Tot.red. & Bus & ot.red \\
\hline \multirow[t]{3}{*}{ (Bus) } & & (No. & $(\%)$ & (No & ) $(\%)$ & (No.) & $(\%)$ \\
\hline & $2-3$ & 1 & 24.43 & 1 & 24.61 & 14 & 62 \\
\hline & $4-9$ & & 2.73 & 3 & 3.82 & 14 & 19 \\
\hline \multirow[t]{3}{*}{14} & $9-10$ & 1 & 2.01 & 1 & 2.05 & 14 & 54 \\
\hline & $2-6$ & 7 & 5.96 & 11 & 6.06 & 30 & 10 \\
\hline & $3-4$ & 13 & 15.19 & 18 & 17.66 & 30 & 22 \\
\hline \multirow[t]{3}{*}{30} & $19-20$ & 1 & 2.12 & 1 & 2.39 & 30 & 66 \\
\hline & $2-3$ & 2 & 1.46 & 5 & 1.55 & 57 & 90 \\
\hline & $30-31$ & 1 & 0.39 & 1 & 0.39 & 57 & 47 \\
\hline \multirow[t]{3}{*}{57} & $54-55$ & 1 & 0.42 & 1 & 0.42 & 57 & 13 \\
\hline & $6-7$ & 1 & 1.21 & 1 & 1.21 & 118 & 65 \\
\hline & $90-92$ & 1 & 0.04 & 1 & 0.07 & 118 & 2 \\
\hline \multirow[t]{3}{*}{118} & $112-116$ & 1 & 0.67 & 1 & 0.68 & 118 & 21 \\
\hline & $3-18$ & 30 & 2.5 & 36 & 2.88 & 300 & 15 \\
\hline & $103-139$ & 1 & 0.7 & 1 & 0.83 & 300 & 26 \\
\hline 300 & $221-224$ & 1 & 0.3 & 2 & 0.62 & 300 & 72 \\
\hline
\end{tabular}




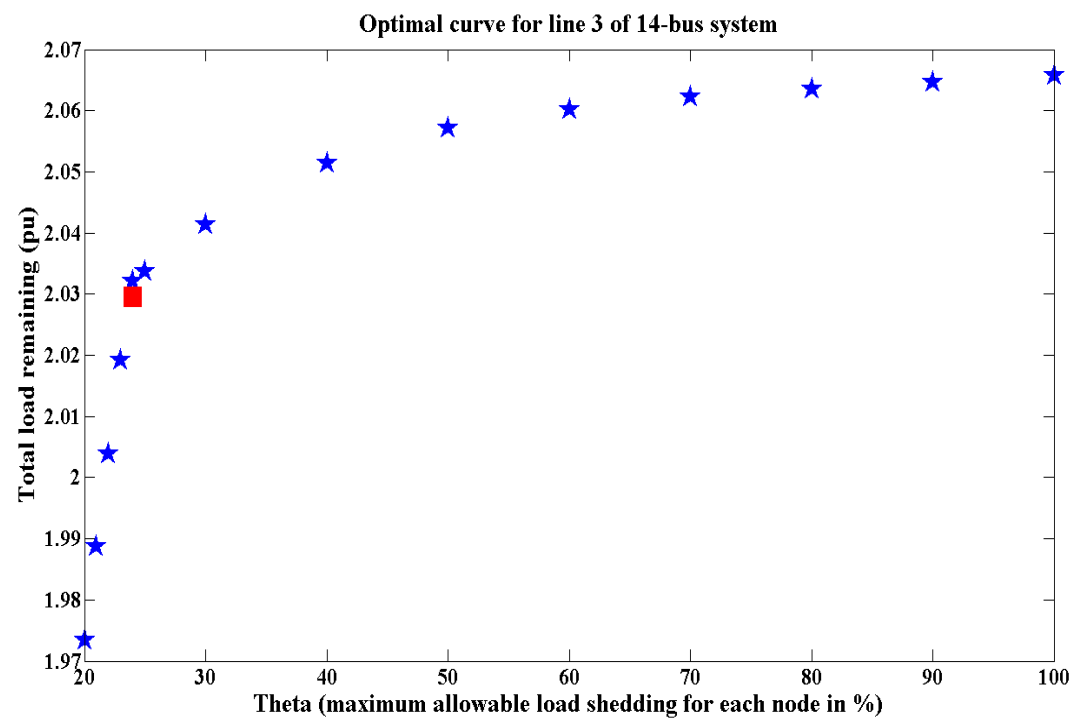

Fig. 1. The optimal curve for line 2 of the 14-bus system and the result of the tree strategy for the same line

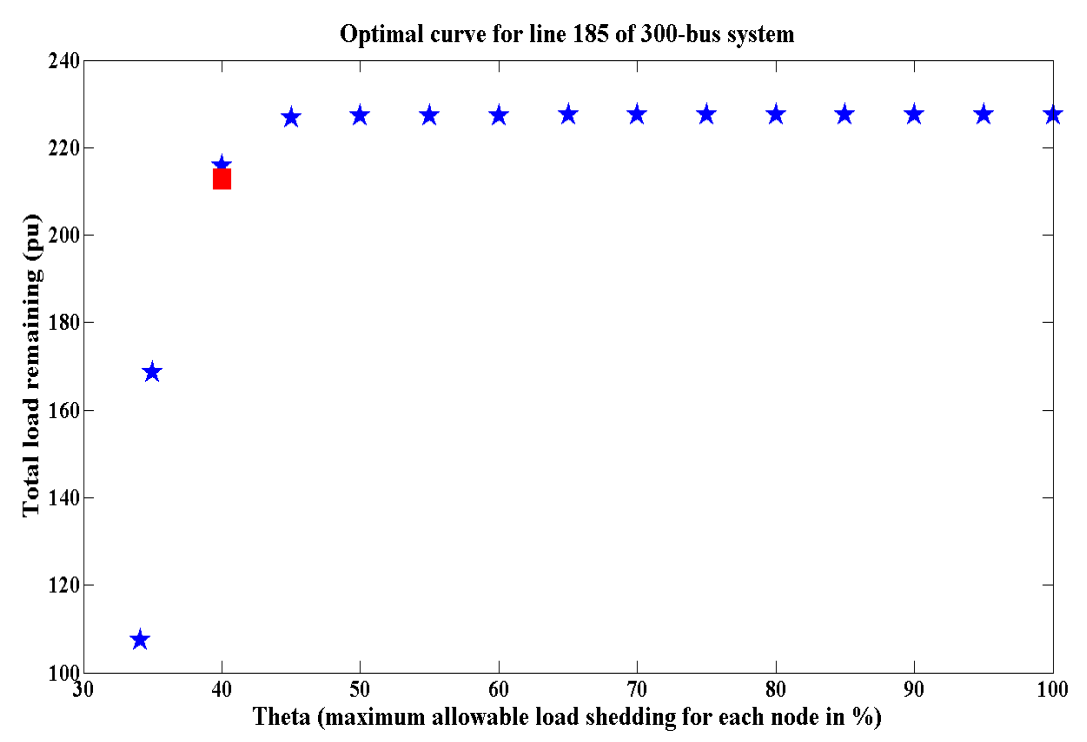

Fig. 2. The optimal curve for line 185 of the 300-bus system and the result of the tree strategy for the same line 


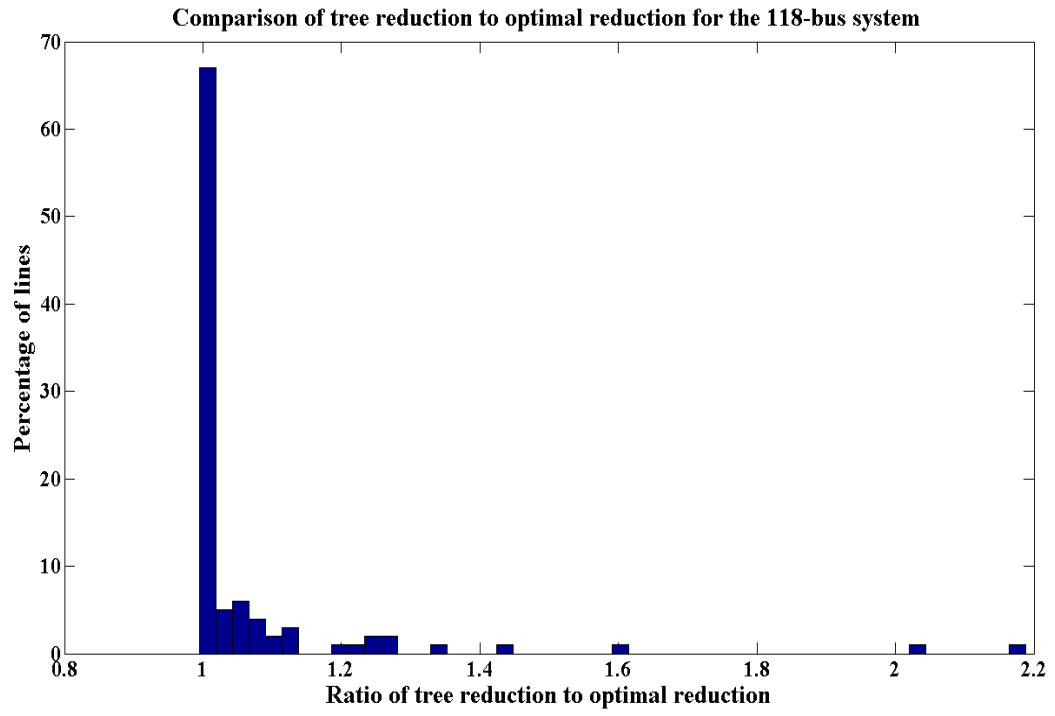

Fig. 3. Histogram representing the ratio of the tree load reduction to the optimal load reduction for all the working cases of the 118-bus system

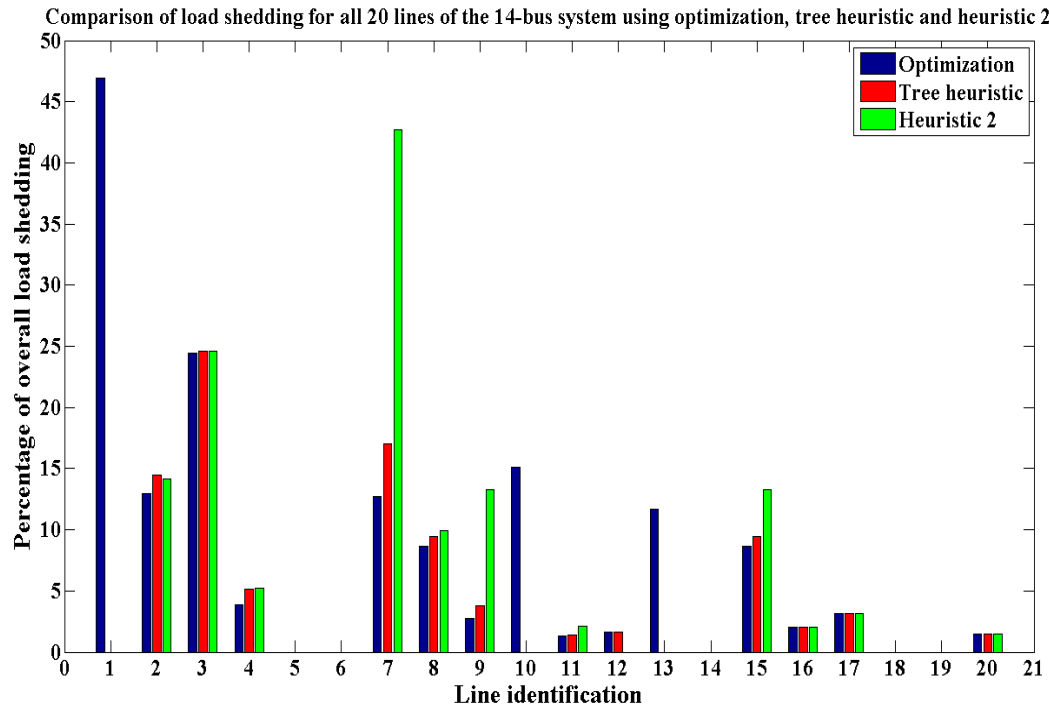

Fig. 4. Comparison between the optimization, tree heuristic and heuristic 2 for the 14-bus system 


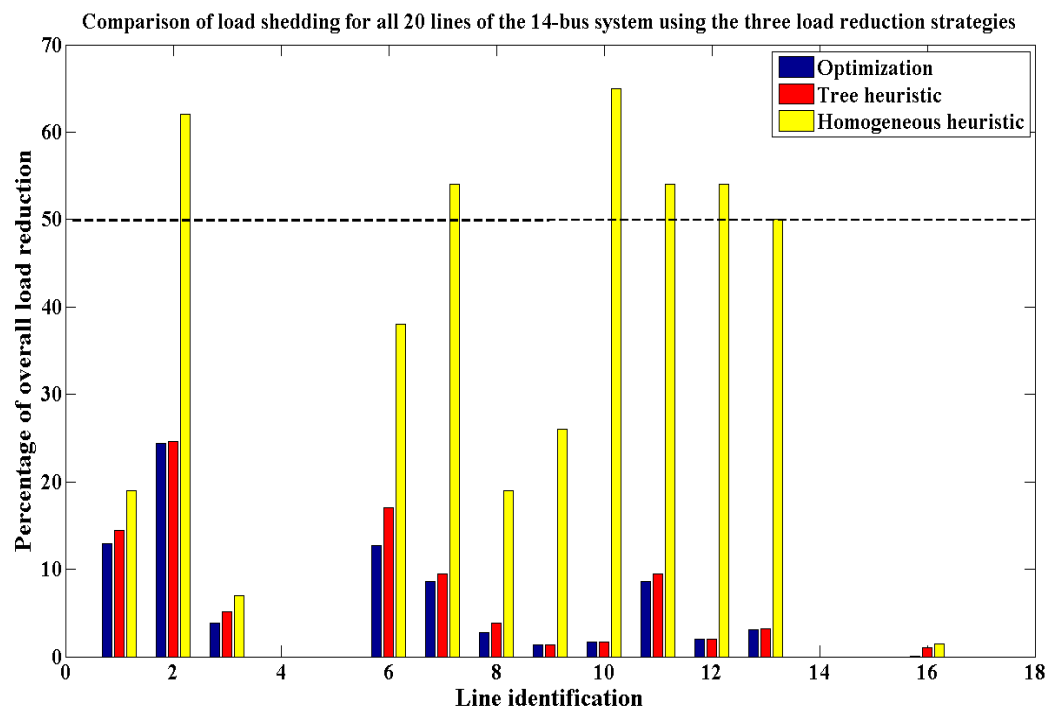

Fig. 5. Comparison of the optimization, tree heuristic and homogeneous load shedding heuristic for all 20 lines of the 14-bus system

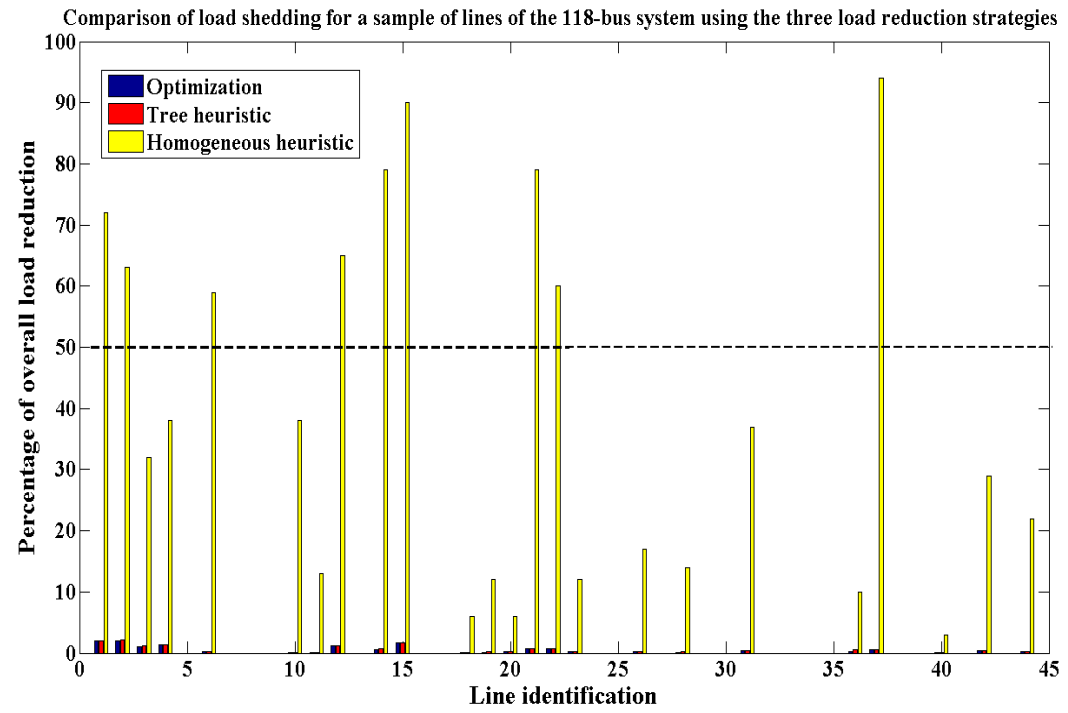

Fig. 6. Comparison of the optimization, tree heuristic and homogeneous load reduction heuristic for a sample of lines of the 118-bus system 


\section{REFERENCES}

[1] North American Electric Reliability Council, "Evaluation of criteria, methods, and practices used for system design, planning, and analysis response to nerc blackout recommendation 13c [online]," http: / / www . nerc . $\mathrm{com} / \mathrm{docs} / \mathrm{pc} / \mathrm{tis} /, 2005$.

[2] P. Kundur, Power System Stability and Control, McGraw-Hill, Inc., 1994.

[3] M. L. Sachtjen, B. A. Carreras, and V. E. Lynch, "Disturbances in a power transmission system," Physical Review E vol.61, no.5 ,4877, May 2000.

[4] B. A. Carreras, D. E. Newman, I. Dobson, and A. B. Poole, "Evidence of self-organized criticality in electric power system blackouts," in Proceedings of the 34th Annual Hawaii International Conference on System Sciences, Maui, Hawaii, USA, January 2001.

[5] J. Glanz and R. Perez-Pena, "90 seconds that left tens of millions of people in the dark," New York Times, August 2003.

[6] P. Fairley, "The unruly power grids," IEEE Spectrum, August 2004.

[7] The Indian Express, “Power blackout hits india again,” July 31.

[8] J. Chen, J. S. Thorp, and I. Dobson, "Cascading dynamics and mitigation assessment in power system disturbances via a hidden failure model," International Journal of Electrical Power and Energy Systems, vol.27, no.4, pp.318-326, May 2005.

[9] J. S. Thorp, A. G. Phadke, S. H. Horowitz, and S. Tamronglak, "Anatomy of power system disturbances: Importance sampling," International Journal of Electrical Power and Energy Systems, vol.20, no.2, pp.147152, February 1998.

[10] D. Xu and A. A. Girgis, "Optimal load shedding strategy in power systems with distributed generation," in Proceedings of the IEEE Power Engineering Society Winter Meeting, Columbus, OH, USA, January 2001.

[11] E. E. Aponte and J. K. Nelson, “Time optimal load shedding for distributed power systems," IEEE Transactions on Power Systems, vol.21, no.1, pp.269-277, February 2006.

[12] H. Bevrani, A. G. Tikdari, and T. Hiyama, "An intelligent based power system load shedding design using voltage and frequency information," in Proceedings of the 2010 International Conference on Modelling, Identification and Control, Okayama, Japan, July 2010.

[13] Washington University, "Power systems test case archive," http://www.ee.washington.edu/ research/pstca/index.htm, 1999.

[14] Y. Zhao, N. Zhou, J. Zhou, K. Xie, Y. Liu, and J. Kuang, "A heuristic approach to local load shedding scheme for reliability assessment of bulk power systems," in Proceedings of the 2005 IEEE/PES Transmission and Distribution Conference and Exhibition: Asia and Pacific, Dalian, China, August 2005.

[15] B. A. Carreras, V. E. Lynch, I. Dobson, and D. E. Newman, "Critical points and transitions in an electric power transmission model for cascading failure blackouts," CHAOS, vol.12, no.4, pp.985-994, September 2002.

[16] P. Hines, K. Balasubramaniam, and E. C. Sanchez, "Cascading failures in power grids," IEEE Potentials, vol.28, no.5, pp.24-30, September 2009.

[17] C. W. Taylor, F. R. Nassief, and R. L. Cresap, "Northwest power pool transient stability and load shedding controls for generation-load imbalances," IEEE Transactions on Power Apparatus and Systems, vol.PAS-100, no.7, pp.3486-3495, July 1981. 
[18] R. Faranda, A. Pievatolo, and E. Tironi, "Load shedding: A new proposal," IEEE Transactions on Power Systems, vol.22, no.4, pp.2086-2093, November 2007.

[19] X. Fu and X. Wang, "Load shedding scheme ensuring voltage stability," in Proceedings of the IEEE Power Engineering Society General Meeting, Tampa, FL, USA.

[20] T. S. P. Fernandes, J. R. Lenzi, and M. A. Mikilita, "Load shedding strategies using optimal load flow with relaxation of restrictions," IEEE Transactions on Power Systems, vol.23, no.2, pp.712-718, May 2008.

[21] J. J. Ford, H. Bevrani, and G. Ledwich, “Adaptive load shedding and regional protection," International Journal of Electric Power Systems Research, vol.31, no.10, pp.611-618, November 2009.

[22] J. Jung, C-C. Liu, S. L. Tanimoto, and V. Vittal, "Adaptation in load shedding under vulnerable operating conditions," IEEE Transactions on Power Systems, vol.17, no.4, pp.1199-1205, November 2002.

[23] H. Seyedi and M. Sanaye-Pasand, "New centralised adaptive load-shedding algorithms to mitigate power system blackouts," IET Generation, Transmission, Distribution, vol.3, no.1, pp.99-114, October 2008.

[24] C. M. Affonso and L. C. P. Da Silva, "Potential benefits of implementing load management to improve power system security," Electrical Power and Energy Systems, vol.32, no.6, pp.704-710, January 2010.

[25] K. Seethalekshmi, S. N. Singh, and S. C. Srivastava, "A synchrophasor assisted frequency and voltage stability based load shedding scheme for self-healing of power system," IEEE Transactions on Smart Grid, vol.2, no.2, pp.221-230, June 2011.

[26] C-R. Chen, W-T. Tsai, H-Y. Chen, C-Y. Lee, C-J. Chen, and H-W. Lan, "Optimal load shedding planning with genetic algorithm," in Proccedings of the 2011 IEEE Industry Applications Society Annual Meeting, Taipei, Taiwan, October 2011.

[27] A. Saffarian and M. Sanaye-Pasand, "Enhancement of power system stability using adaptive combinational load shedding methods," IEEE Transactions on Power Systems, vol.26, no.3, pp.1010-1020, August 2011.

[28] M. Q. Ahsan, A. H. Chowdhury, S. S. Ahmed, I. H. Bhuyan, M. A. Haque, and H. Rahman, "Technique to develop auto load shedding and islanding to prevent power system blackout," IEEE Transactions on Power Systems, vol.27, no.1, pp.198-205, February 2012.

[29] T. J. Overbye, X. Cheng, and Y. Sun, "A comparison of the ac and dc power flow models for lmp calculations," in Proceedings of the 37th Annual Hawaii International Conference on System Sciences, Big Island, Hawaii, USA, January 2004.

[30] K. Purchala, L. Meeus, D. Van Dommelen, and R. Belmans, "Usefulness of dc power flow for active power flow analysis," in Proceedings of IEEE Power Engineering Society General Meeting, San Fransisco, CA, USA, June 2005.

[31] D. Van Hertem, J. Verboomen, K. Purchala, R. Belmans, and W. L. Kling, "Usefulness of dc power flow for active power flow analysis with flow controlling devices," in The 8th IEE International Conference on AC and DC Power Transmission,Savoy Place, London, UK, July 2006.

[32] B. R. Gungor, Power Systems, Technology Publications, 1988.

[33] J. D. Glover, M. S. Sarma, and T. J. Overbye, Power System Analysis and Design, Cengage Learning.

[34] N. Fan, D. Izraelevitz, F. Pan, P. M. Pardalos, and J. Wang, "A mixed integer programming approach for optimal power grid intentional islanding," Energy Systems, vol.3, no.1, pp.77-93, January 2012. 